\title{
Synchronous Power Controller for Distributed Generation Units
}

\author{
Andrés Tarrasó \\ Universitat Politecnica de Catalunya \\ SEER (UPC) \\ Terrassa, Spain \\ andres.tarraso@upc.edu \\ Jose Ignacio Candela \\ Universitat Politecnica de Catalunya \\ SEER (UPC) \\ Terrassa, Spain \\ candela@ee.upc.edu
}

\author{
Cristian Verdugo \\ Universitat Politecnica de Catalunya \\ SEER (UPC) \\ Terrassa, Spain \\ andres.tarraso@upc.edu \\ Pedro Rodriguez \\ Universidad Loyola Andalucia \\ LoyolaTech \\ Sevilla, Spain \\ prodriguez@uloyola.es
}

\author{
Ngoc Bao Lai \\ Universidad Loyola Andalucia \\ Loyolatech \\ Sevilla, Spain \\ nblai@uloyola.es
}

\begin{abstract}
Renewable energies has shown to be a promising solution to improve the energy injected to the electrical network. However, the lack of inertia in such systems have proven to affect the grid stability, as those technologies operate as current controlled sources they not provide grid support functionalities. This issue has fostered the implementation of new control strategies to enable power plants to provide a coordinated action to support some inertia and damping into the system. Synchronous controllers have proven to be an excellent option for distributed renewable generation, which allows to emulate the inertia of a synchronous generators, having a better interaction between the power converter and the electrical grid. However, the implementation of such control strategies require power converter to be smarter and include advanced control algorithms. In this paper, a new control strategy to control different distributed generation units to have a coordinated action in front of the electrical grid is presented, reducing the cost of the power converter by extracting the synchronous control from the internal controller and replacing in by a central synchronous controller.
\end{abstract}

Keywords-Renewable energy, synchronous control, power converter

\section{INTRODUCTION}

During the last decade, grid integration of renewable energies has been increasing significantly, mainly due to reduction of the cost associated to PV and wind power. However, this scenario implies the appearance of other drawbacks, as the overall inertia decrease in electrical networks with a high share of these technologies. With less inertia the grid stability becomes weaker and less stable. Therefore, there is a high interest in reaching solutions able to provide some inertia in renewable energies system, that would make compatible the development of green energies with grid stability in the long run. Several works have presented solutions that could enhance a friendly renewable energy integration to the electrical grid, as a sample some control strategies, topologies and components were presented in [1], although there is still a big number of other references dealing with these topics. As power levels in PV and wind energy grow, the problems related to the large scale integration into the electrical network, such as stability also increase [2][3][3][3][3]. Due to this, several grid supporting functionalities have been added to such technologies in the last years. This is the case of reactive power profiles demanded by grid codes, which are oriented to support the grid under voltage sags [4]. On the other hand, frequency support depends on the active power balance during frequency events at the point of common coupling (PCC) [5]. As the penetration of wind and PV power generation increases, new demands and requirements arise. However, each new challenge comes together with a partial solution provided by the industry.

There are many researchers studying the effect of the cumulative addition of grid support controllers at each power conversion unit of power plants, as well as its effect at the PCC. Lately, different approaches of the virtual synchronous generation concept have emerged and its implementation in single power conversion units as well as its performance in hundreds of units working in parallel are being studied [6]. However, even if the synchronous control is implemented in a distributed manner, it is not possible to coordinate a joint action of power converters at the PCC using classical power plant controllers. Due to this, this paper introduces how the traditional synchronous power control can be modified to control distributed generation units as a unique element connected to the grid. The synchronous power control has the capability of adapting its performance depending on the grid conditions. It is responsive to frequency and voltage faults and it is able to work seamlessly in resistive or inductive grids. Likewise, the synchronous power control is able to work either in the grid connected and islanded operation mode [7]. Although, this control concept has many implementations, the main goal behind all of them is the same, the inertia emulation and its improved response during grid transients. On the one hand, there is the approach of the so called Synchronous Power Controller (SPC) [8], which emulates the electromechanical equations of a synchronous generator with configurable inertia and damping, and by terms of a virtual admittance emulates the stator of the system [9]. On the other hand, there are other solutions which have different implementation, such as the full representation of the synchronous machine [10], or the utilization of a simple version through its swing equation [11] or the emulation of inertia and damping of the generator in addition to the natural impedance parameters of the stator [12].

In this paper, a proposal for a synchronous control capable of grouping different power converter units into a unique emulated synchronous generator is going to be presented. This work proposes a synchronous control capable of performing with most traditional power controllers inside the power converter, to provide a joint action at the PCC of the system. 


\section{SyNCHRONOUS POWER CONTROL}

Different synchronous generator control algorithms have been presented in the literature, which try to emulate the behavior of the synchronous generator during transient and steady state performance. However, this work emphasizes in the so called Synchronous Power Controller (SPC) presented in [7] and [8]. This control strategy is based in the emulation of the swing equation and a virtual admittance, which provides not only the capacity of controlling the fundamental frequency, but also harmonic frequencies as presented in [13]. These features allow converters to provide similar or enhanced characteristics of a synchronous generators in terms of grid support functionalities no matter the grid characteristics.

The SPC controller lays on the capability to control the power converter as a controlled current source, where unlike other virtual synchronous machine implementation which uses the power converter as a controlled voltage source, which tends to be more unstable depending on the grid scenario. In this regard, the difference between the voltage measured at the point of common coupling (PCC) of the power converter, $v_{c}$, is subtracted from a virtual voltage representing the electromotive force of the synchronous generator, $e_{v}$. Generating in this manner a voltage difference which generates the current reference through a virtual admittance block, as presented in Fig. 1.
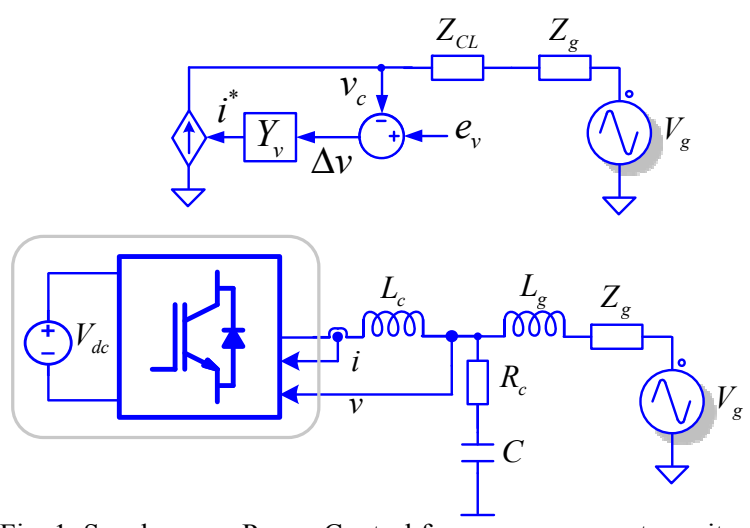

Fig. 1: Synchronous Power Control for a power converter unit

The electromechanical interaction to the electrical grid is based on the traditional synchronous generator behavior. Where the system is synchronized via the power balance between its electromotive force and the grid voltage [15]. This action is generated by the swing equation which represents the mechanical characteristic of the generator, which can be represented as (1), where the torque $\Gamma$ depends on the angular acceleration of the rotor $\omega_{r}$. In this manner, the swing equation is able to generate the synchronization system through the power balance of the power converter.

$$
\Gamma=J \cdot \frac{d \omega_{r}}{d t}+D \Delta \omega
$$

The angular position of the internal electromotive force (EMF) is generated by the swing equation, in which the $J$ defines the constant of inertia of the system and the $\omega_{r}$ is responsible of accelerate or decelerate the angular speed of the system to maintain the system synchronous to the electrical grid. Additionally, the D parameter is included into the swing equation which adds a damping factor into the system. This term represent the damping windings included into traditional synchronous generators.

The small signal analysis of the system can be transformed into the power balance between the mechanical power in the virtual shaft and the electrical power at the output, defining equation (2).

$$
P_{m}-P_{e}=\omega_{g}\left(J \frac{d \omega_{r}}{d t}+D \Delta \omega\right)
$$

By terms of using equation (2) the block diagram can be developed as presented in Fig. 2.

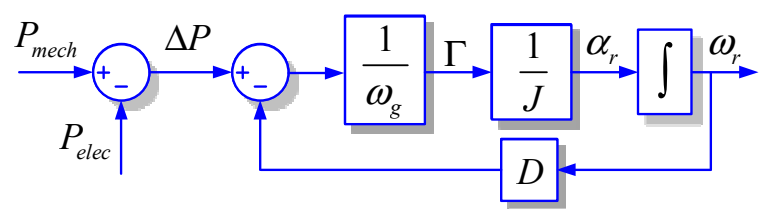

Fig. 2: Mechanical small signal model of a synchronous generator

This block is integrated into the power controller diagram as the Power-Loop Controller (PLC) which is in charge of generating the rotor speed to synchronize the system to the electrical grid.

The virtual phase of the system is obtained through the integration of the rotor speed of the system, $\omega_{r}$. This phase angle determines the EMF angular speed and phase, imposing the $e(t)$ vector. In order to inject zero active and reactive power, the phase and magnitude of the virtual voltage $e(t)$ has to be equal to the grid voltage $v(t)$. This leads to the power regulation where a change in phase angle will determine the amount of active power and a movement in voltage magnitude will generate reactive power. The PLC controller is in charge of regulating the active power balance in the system, as presented in the previous electromechanical description. For the reactive power regulation, a PI controller is used to determine the peak magnitude of the virtual voltage $e(t)$.

The electromagnetic interaction is determined by the virtual admittance block, which has to do with the amount of current injected to the PCC. The equivalent transfer function of the virtual admittance can be determined as (3).

$$
Y_{V}(s)=\frac{1}{R_{V}+s L_{V}}
$$

The virtual admittance has a natural proportional relationship between the amount of current injected to the electrical grid by the difference of the virtual voltage and the voltage at the grid. The block generates a current reference which respond to any grid voltage disturbance, which will be generating new current references depending on the grid voltage status. Which provide an instantaneous response during grid voltage transients and disturbances. 


\section{SYNCHRONOUS POWER CONTROL FOR DISTRIBUTED UNITS}

Previous works have studied the synchronous power controller in different scenarios [13]. Renewable power plants are defined by different distributed generators connected to the same PCC, and the active and reactive power regulation is performed by TSO, which specifies the requirements for active and reactive power, as well as the frequency and voltage droop regulation to provide grid support functionalities. The purpose behind the synchronous control for distributed generators concept is to emulate the synchronous generator in an entire power plant and operate all converters as a single unit, Fig. 3. In this way, from the grid point of view, the power plant will be able to provide frequency and voltage support as a unique synchronous machine, where all power converters will be coordinated to provide energy to the grid according to the power level available. The synchronous control block provides the references to each converter

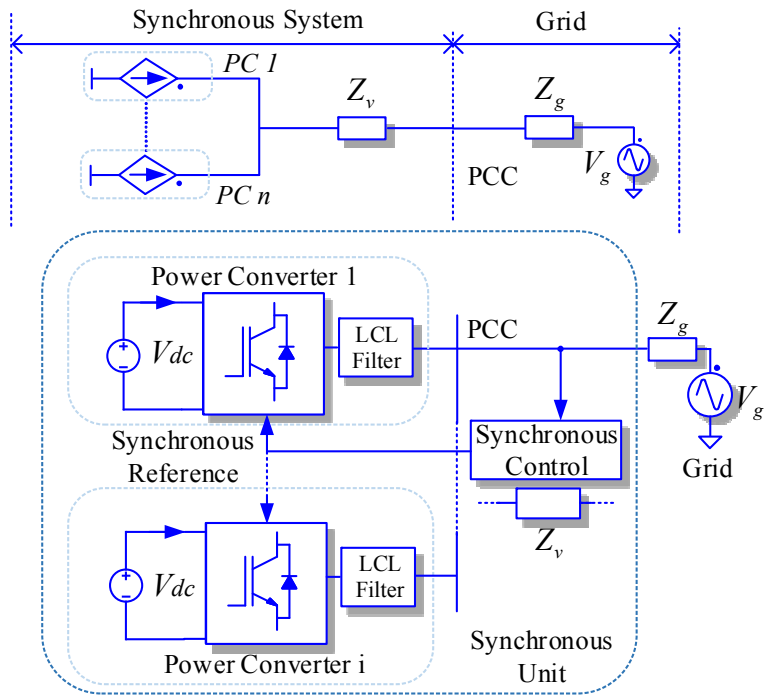

Fig. 3: Synchronous Control for multiple power converters

The synchronous control proposed is a control strategy which provides synchronous power references to each converter according to the grid requirements at the $\mathrm{PCC}$ and the maximum power available in the power plant. Based on the SPC, the mechanical control is designed to generate the load angle and the voltage amplitude $E$, which are specified by the inertia and the damping emulation. Subsequently, the virtual voltage amplitude $E$ and the load angle $\delta$ are used to calculate the synchronous power reference required by the power plant through the instantaneous power flow expression presented in (3) and (4). According to [14], these equations represent the power sharing between two ac sources.

$$
\begin{aligned}
& P_{s y n c, i}=\frac{k_{i} V}{R^{2}+X^{2}}[R(V-E \cos (\delta))+X E \sin (\delta)] \\
& Q_{s y n c}=\frac{V}{R^{2}+X^{2}}[-R E \sin (\delta)+X(V-E \cos (\delta))]
\end{aligned}
$$

Where $E$ and $V$ are the amplitude of the virtual voltage and the grid voltage respectively, $R$ and $X$ are the parameters of the virtual impedance and $\delta$ is the load phase angle between the virtual voltage $e$ and $v$. Since the load angle $\delta$ depends on the voltage difference, it is possible to obtain this angle through the virtual voltage provided by the mechanical part of the synchronous control. This is accomplished through the swing equation implemented in the power loop controller, where an angle $\theta_{\text {sync }}$ obtained from the control loop is used to rotate the grid voltage in the $d q$ frame reference and then used to calculate the load angle by the inverse tangent function, the full control schematic is presented in Fig. 4.

All power converters are controlled with the synchronous active and reactive power provided by the SGC. The internal control of each converter has to be able to adjust the power reference into the current reference. This adjustment is performed through the expression (5) and (6) where a $d q$ or an $\alpha \beta$ components can be used to set the current references. The current reference transform will define the requirement of a PI or a proportional-resonant (PR) controller for the current regulation.

$$
\begin{aligned}
& i_{\alpha}^{*}=\frac{P_{r e f} v_{\alpha}+Q_{r e f} v_{\beta}}{v_{\alpha}^{2}+v_{\beta}^{2}}, i_{d}^{*}=\frac{P_{r e f} v_{d}+Q_{r e f} v_{q}}{v_{d}^{2}+v_{q}^{2}} \\
& i_{\beta}^{*}=\frac{P_{r e f} v_{\beta}-Q_{r e f} v_{\alpha}}{v_{\alpha}^{2}+v_{\beta}^{2}}, i_{q}^{*}=\frac{P_{r e f} v_{q}-Q_{r e f} v_{d}}{v_{d}^{2}+v_{q}^{2}}
\end{aligned}
$$

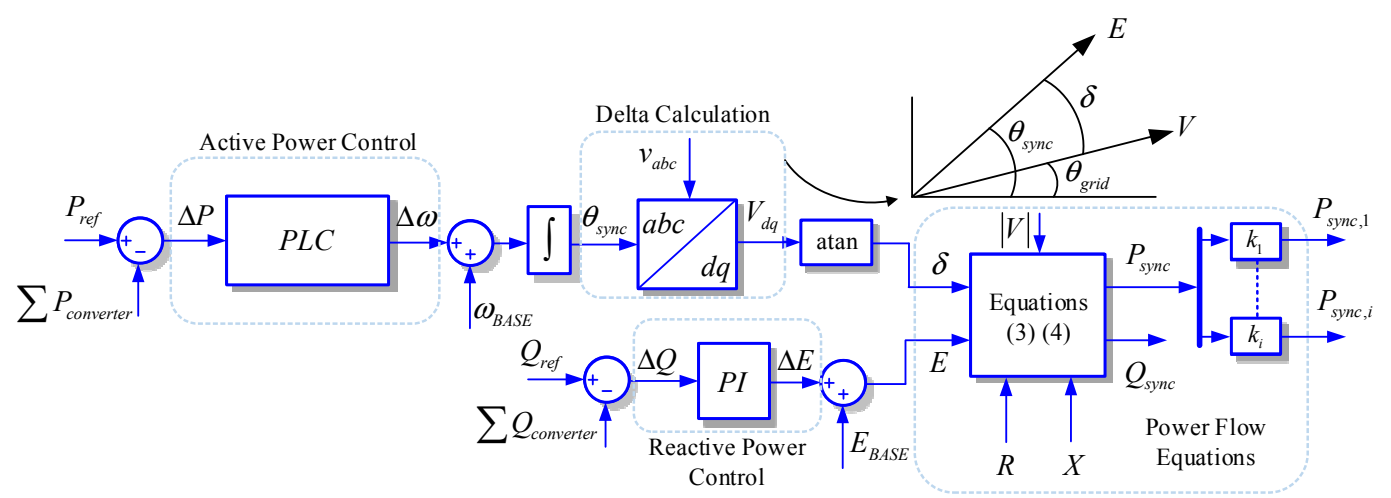

Fig. 4: Synchronous Grid controller scheme 


\section{Simulation RESUlTS}

The simulation setup is formed by two power converter connected to the electrical grid, Fig. 5. The nominal power of the converters is $10 \mathrm{kVA}$, and they are connected to an inductive line with a short circuit ratio (SCR) of 5 . The parameters of the power converter are specified in Table. 1, as well as the grid parameters for inductance.

\begin{tabular}{|c|c|}
\hline Nominal Power & $10 \mathrm{kVA}$ \\
\hline Switching Frequency & $10 \mathrm{kHz}$ \\
\hline Converter side Inductor & $2.6 \mathrm{mH}$ \\
\hline Capacitor & $5.5 \mathrm{uF}$ \\
\hline Grid side inductor & $0.76 \mathrm{mH}$ \\
\hline Grid resistance & $0.3184 \Omega$ \\
\hline Grid Inductance & $10 \mathrm{mH}$ \\
\hline
\end{tabular}

Table. 1: Inverter and grid parameters of the simulation

The simulations results are focused on highlighting the performance of the central synchronous controller when coordinating the performance of power converters, enabling those to perform as part of the synchronous generator by injecting the necessary active and reactive power to the electrical grid. The tests are selected to demonstrate the capability of the system to distribute power among power converters as well as to provide support on voltage during grid disturbance. On the one hand, active power injection with different power distribution of power among converters will be presented, as well as a disconnection of one of the power conversion units to highlight the capability of the system to withstand a sudden disconnection within the control area. On the other hand, one of the most demanding grid faults will be generated in the system, a voltage sag, forcing the power converters to provide a joined action at the PCC in order to restore the voltage.

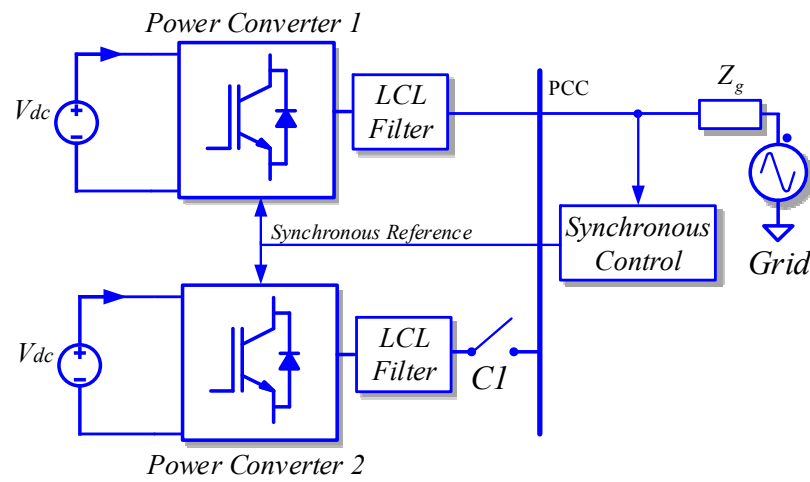

Fig. 5: Schematic of the simulation setup composed by two power converter connected to the electrical grid.

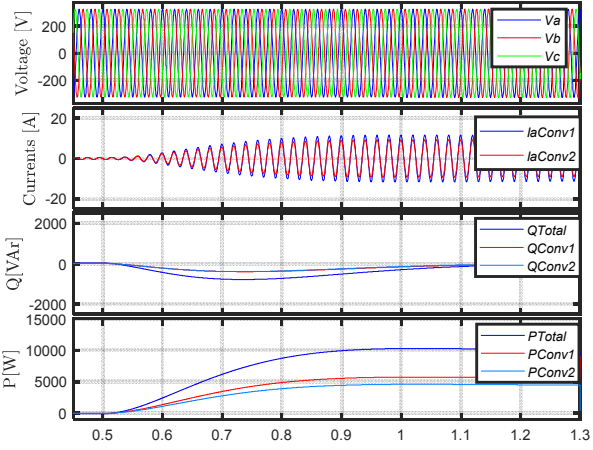

a)

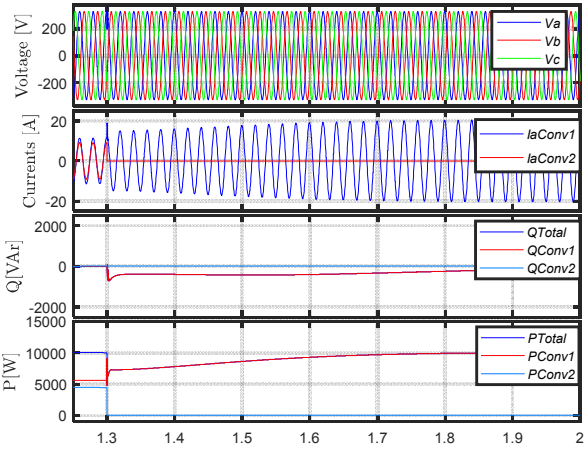

b)

Fig. 6: Central synchronous controller generating references for two power converters a) Power step to $10 \mathrm{~kW}$ with different power reference gain values. b) Disconnection of converter 2 during power injection to the grid.

In the first test, the synchronous controller is set to provide $10 \mathrm{~kW}$ at the $\mathrm{PCC}$, where the power converter 1 power distribution gain value is set to a value of $1 \mathrm{p} . \mathrm{u}$ and power converter 2 gain value is set to a value of 0.8 p.u. Fig. 6a presents the power step of the coordinated synchronous system during a power step to $10 \mathrm{~kW}$. The power converters are injecting active power according to the gain value set to each power converter, power converter 1 is injecting $5.55 \mathrm{~kW}$ and the power converter 2 is injecting $4.45 \mathrm{~kW}$. Fig. $6 \mathrm{~b}$ presents a disconnection of the power converter 2 , the central synchronous control is able to read that the power has been reduced, which force the value of the mechanical controller to increase the phase angle $\delta$ in the system, trying to inject more power to restore the power lost at the PCC by sending additional power reference to the power converter 1 .

Un the second test, the low voltage ride through (LVRT) capacity is tested, where a balanced voltage sag is generated at the PCC. During the grid fault the power converter has to inject reactive power to help the restoration of the voltage level. Fig. 7a presents the LVRT performance of the converter where converter 1 and 2 have the same constant gain $k$ for power distribution among units. Therefore, the same response and reactive power will be obtained during the voltage fault by the power converters. In Fig. $7 \mathrm{~b}$ the same LVRT test is conducted, however in this case the gain value of the converter 2 is set to $0.8 \mathrm{p}$.u, which forces converter 1 to inject more reactive power and converter 2 to inject less reactive power to the grid. 


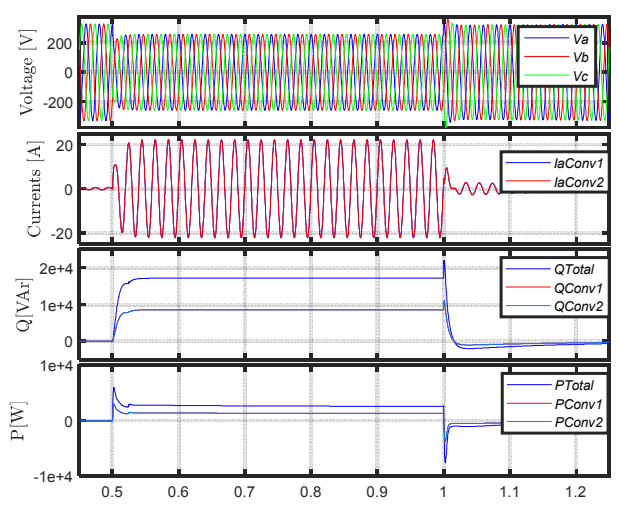

a)

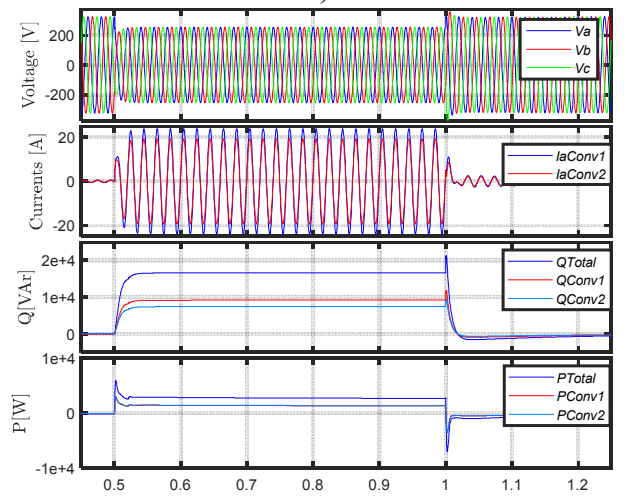

b)

Fig. 7: Central synchronous control under LVRT test. a) Coordinated action during a voltage sag with equal parameters for the reactive power gain. b) Coordinated action during a voltage sag with different parameters for the reactive power gain.

As a conclusion, if any of the units included inside of the synchronous control is disconnected or the gain values of the power controller are modified the synchronous central controller will decrease or increase its delta value to generate the needed synchronous power reference to the remaining power converters.

\section{CONCLUSIONS}

In this paper the synchronous control for distributed generation units has been presented. This controller is able to provide synchronous power references different power converters within its control system and share between units the power reference to provide a unique performance at the PCC. This central synchronous controller takes advantage of the classical SPC controller to generate the load angle, which determines the amount of power being exchanged between voltage sources through an impedance. Allowing the system to control the power converter units as a part of a synchronous system, injecting active or reactive power depending on the $\delta$ parameter, and the voltage difference between the voltage $V$, at the grid side, and the virtual voltage $E$. The synchronous control has been validated in simulation.

The system uses the SPC controller to generate its phase angle, generating the delta control, which determines the amount of power being exchanged between voltage sources through an impedance. Forcing the power converter units inside of the synchronous control to inject active or reactive power depending on the $\delta$ parameter, and the voltage difference between the voltage $\mathrm{V}$, at the grid side, and the virtual voltage $\mathrm{E}$. Which regulates the amount of power of the power converter.

\section{ACKNOWLEDGMENT}

This work was supported by the European Commission under project FLEXITRANSTORE - H2020-LCE-2016-2017SGS-774407 and by the Spanish Ministry of Science Innovation and Universities under the project FLEXIGRID RTI2018-100921-B-C21. Any opinions, findings and conclusions or recommendations written in this work are those of the authors and do not necessarily reflect those of the host institutions and funders.

\section{REFERENCES}

[1] A. K. Cabrera, H. U. Banna, C. Koch-Ciobotarus, and S. Ghosh, "Optimization of an air conditioning unit according to renewable energy availability and user's comfort," in IEEE PES Innovative Smart Grid Technologies, Europe, 2014, pp. 1-7.

[2] J. Widén, E. Wäckelgård, J. Paatero, and P. Lund, "Impacts of distributed photovoltaics on network voltages: Stochastic simulations of three Swedish low-voltage distribution grids," Electr. Power Syst. Res., vol. 80, no. 12, pp. 1562-1571, 2010.

[3] J. V Paatero and P. D. Lund, "Effects of large-scale photovoltaic power integration on electricity distribution networks," Renew. Energy, vol. 32, no. 2, pp. 216-234, 2007.

[4] J. A. D. de Leon, B. Kehrli, and A. Zalay, "How the Lake Bonney wind farm met ESCOSA's, NEMMCO's, and ElectraNet's rigorous interconnecting requirements," 2008 IEEE/PES Transmission and Distribution Conference and Exposition. pp. 1$5,2008$.

[5] E. D. Dinu, D. Ilişiu, I. Făgărăşan, S. S. Iliescu, and N. Arghira, "Voltage \&\#x2014; Reactive power control in renewables power plants: Technical requirements applied in the Romanian power grid," 2016 IEEE International Conference on Automation, Quality and Testing, Robotics (AQTR). pp. 1-5, 2016.

[6] D. Remon, A. M. Cantarellas, and P. Rodriguez, "Equivalent Model of Large-Scale Synchronous Photovoltaic Power Plants," IEEE Trans. Ind. Appl., vol. 52, no. 6, pp. 5029-5040, 2016.

[7] P. Rodriguez, C. Citro, I. Candela, J. Rocabet, and A. Luna, "Flexible grid connection and islanding of SPC-based PV power converters," Energy Conversion Congress and Exposition (ECCE), 2015 IEEE. pp. 450-459, 2015.

[8] W. Zhang, A. M. Cantarellas, J. Rocabert, A. Luna, and P. Rodriguez, "Synchronous Power Controller With Flexible Droop Characteristics for Renewable Power Generation Systems," IEEE Trans. Sustain. Energy, vol. 7, no. 4, pp. 1572-1582, Oct. 2016.

[9] P. Rodriguez, I. Candela, and A. Luna, "Control of PV generation systems using the synchronous power controller," 2013 IEEE Energy Conversion Congress and Exposition. pp. 993-998, 2013.

[10] Y. Chen, R. Hesse, D. Turschner, and H. Beck, "Dynamic Properties of the Virtual Synchronous Machine ( VISMA )," Proc. ICREPQ, pp. 1-5, 2011.

[11] K. Sakimoto, Y. Miura, and T. Ise, "Stabilization of a power system with a distributed generator by a Virtual Synchronous Generator function," in 8th International Conference on Power Electronics - ECCE Asia, 2011, pp. 1498-1505.

[12] S. D'Arco, J. A. Suul, and O. B. Fosso, "A Virtual Synchronous Machine implementation for distributed control of power converters in SmartGrids," Electr. Power Syst. Res., vol. 122, pp. 180-197, 2015.

[13] A. Tarrasó, J. I. Candela, J. Rocabert, and P. Rodriguez, "Grid voltage harmonic damping method for SPC based power converters with multiple virtual admittance control," in 2017 IEEE Energy Conversion Congress and Exposition (ECCE), 2017, pp. 64-68.

[14] N. J. Kundur, P. and Balu, Power System Stability and Control. McGraw-Hill, 1994. 